\title{
Plasma metabolomics reveals a potential panel of biomarkers for early diagnosis in acute coronary syndrome
}

\author{
Carlos M. Laborde $\cdot$ Laura Mourino-Alvarez • María Posada-Ayala • \\ Gloria Alvarez-Llamas • Manuel Gómez Serranillos-Reus • José Moreu • \\ Fernando Vivanco $\cdot$ Luis R. Padial • María G. Barderas
}

Received: 15 May 2013/ Accepted: 15 October 2013/Published online: 26 October 2013

(C) Springer Science+Business Media New York 2013

\begin{abstract}
Discovery of new biomarkers is critical for early diagnosis of acute coronary syndrome (ACS). Recent advances in metabolomic technologies have drastically enhanced the possibility of improving the knowledge of its physiopathology through the identification of the altered metabolic pathways. In this study, analyses of peripheral plasma from non-ST segment elevation ACS patients and healthy controls by gas chromatography-mass spectrometry (GC-MC) permitted the identification of 15 metabolites with statistical differences $(p<0.05)$ between experimental groups. Additionally, validation by GC-MC and
\end{abstract}

C. M. Laborde · L. Mourino-Alvarez · M. G. Barderas ( $\square)$ Department of Vascular Physiopathology, Hospital Nacional de Paraplejicos, SESCAM, Toledo, Spain

e-mail: megonzalezb@sescam.jccm.es

M. Posada-Ayala · G. Alvarez-Llamas · F. Vivanco Department of Immunology, IIS-Fundación Jiménez Díaz, Madrid, Spain

M. G. Serranillos-Reus

Department of Biochemistry, Hospital Virgen de la Salud, SESCAM, Toledo, Spain

J. Moreu

Department of Hemodynamic, Hospital Virgen de la Salud, SESCAM, Toledo, Spain

F. Vivanco

Department of Biochemistry and Molecular Biology I, Facultad de Biología, UCM, Madrid, Spain

L. R. Padial

Department of Cardiology, Hospital Virgen de la Salud, SESCAM, Toledo, Spain

M. G. Barderas

Proteomic Unit, Hospital Nacional de Paraplejicos, SESCAM,

Toledo, Spain liquid chromatography-MC permitted us to identify a potential panel of biomarkers formed by 5-OH-tryptophan, 2-OH-butyric acid and 3-OH-butyric acid. This panel of biomarkers reflects the oxidative stress and the hypoxic state that suffers the myocardial cells and consequently constitutes a metabolomic signature of the atherogenesis process that could be used for early diagnosis of ACS.

Keywords Metabolomics $\cdot$ Metabolite $\cdot$ Biomarker . Acute coronary syndrome

\section{Introduction}

Cardiovascular diseases (CVD) remain the leading cause of death in developed countries and are expected to become so in emerging countries in the next years (Roger et al. 2011; Bassand et al. 2007). Acute coronary syndromes (ACS) are one of their most prevalent manifestations and include a wide range of clinical presentations, from unstable angina without myocardial necrosis to ST segment elevation myocardial infarction (Chew et al. 2005). It is well recognized that the myocardial under perfusion due to the rupture or erosion of the atherosclerotic plaque constitutes the pathophysiological origin of ACS (Bassand et al. 2007).

Despite the mechanisms underlying the formation of the atherosclerotic plaque and its progression are not completely known, it is well established that the initial stage of the atherogenesis process is characterized by thickening of the arterial wall resulting from the build-up of fatty materials, macrophages and muscular cells (Hackam and Anand 2003; Wilson 2008; Libby et al. 2002; Hansson 2005). There are uncertainties about the definition of plaque instability and the availability of trustworthy specific 
markers to identify plaques prone to rupture in vivo. However, it is generally assumed that plaque instability is caused by a substantial increase in proteolytic activity and inflammatory state (Shen et al. 2010; van Olffen et al. 2010)

From a clinical perspective, its silently and very progressive development through decades constitutes the greatest trouble for the diagnosis of ACS. Unfortunately, the symptoms only become evident when the disease is in an advanced and irreversible state. These shortcomings in the early diagnosis of ACS indicate the need for new biomarkers with real clinical value to predict the disease and establish a stratification of individual cardiovascular risk.

Metabolomics is the systematic study of the complete set of metabolites of a cell, tissue, or organ with the final objective to describe its phenotype (Oliver et al. 1998; Fiehn et al. 2000; Tweeddale et al. 1998; Waterman et al. 2010). The qualitative and quantitative evaluation over time of a large number of metabolites, in easily accessible biological fluids, can supply the description of the biochemical state of an organism, providing valuable information on the interrelations between the various metabolic processes that define this state (van der Greef et al. 2007).

In this sense, plasma and urine are the two more rich metabolite-containing biological fluids and the most interesting samples from a clinical perspective (Kind et al. 2007; Salek et al. 2007; Boernsen et al. 2005). Among the different mass spectrometry based technologies gas chromatography-mass spectrometry (GC/MS) constitutes an excellent platform for targeted and untargeted metabolomics thanks to it sigh sensitivity, great separation capability and the existence of extensive mass spectral libraries (Pasikanti et al. 2008). For the identification of potential biomarkers, the use of statistical software and multivariate analysis permits evaluate specific variations of metabolites that describe the effect of a disease in the different parts of the organism, showing interconnections and interdependences of multiple metabolites (Kemsley et al. 2007).

\section{Materials and methods}

\subsection{Patient selection}

Blood samples of non-ST segment elevation ACS (NSTEACS) patients $(n=35)$ and sex and age matched healthy subjects $(n=35)$ were recruited from the Cardiology Service of Hospital Virgen de la Salud (Toledo, Spain). In case of NSTEACS patients, the sample was obtained in the onset of the syndrome $(t=0 \mathrm{~h})$ defined as the moment when the coronary event is diagnosed. All subjects included in the study were screened with detailed medical history, physical examination and biochemical profile (Table 1). This study was carried out in accordance with the recommendations of the Helsinki Declaration and it was approved by the ethics committee at the Hospital Virgen de la Salud. Signed informed consent was obtained from all subjects prior to their inclusion in the study.

\subsubsection{Plasma isolation}

Five milliliters of blood were collected in sterile EDTA containers (BD Microtainer ${ }^{\circledR}$ ) and samples were immediately taken to our laboratory to prevent sample degradation $(<2 \mathrm{~h})$. Finally, samples were centrifuged at $3,500 \times g(5810 \mathrm{R}$, Eppendorf) for $10 \mathrm{~min}$ at room temperature and the resulting plasma was aliquoted in batches of $500 \mu \mathrm{l}$ and stored at $-80{ }^{\circ} \mathrm{C}$ until GC/MS analysis.

\subsection{Plasma metabolomic profiling}

\subsubsection{Chemicals and reagents}

The N,O-bis (trimethylsilyl) trifluoroacetamide with $1 \%$ trimethylchlorosilane (BSTFA+1 \% TMCS) was obtained from Supelco. Methoxiamine hydrochloride, acetonitrile, methyl pentadecanoate (internal standard, IS) and pyridine were obtained from Sigma-Aldrich. Ultrapure milliQ water was produced by a Milli-Q Reagent Water System.

Table 1 Baseline characteristics of participants

\begin{tabular}{lll}
\hline & $\begin{array}{l}\text { Healthy controls } \\
(n=35)\end{array}$ & $\begin{array}{l}\text { NSTEACS patients } \\
(n=35)\end{array}$ \\
\hline Personal data & & \\
Age \pm SD (years) & $64.2 \pm 11.5$ & $68.3 \pm 9.8$ \\
Sex (male/female) & $17 / 18$ & $27 / 8$ \\
Cardiovascular risk factors & & \\
Smokers & 3 & 10 \\
Ex-smokers & 3 & 8 \\
DMI & 1 & 5 \\
Dyslipidaemia & 6 & 23 \\
Hypertensión & 14 & 22 \\
Renal disease & 0 & 3 \\
Biochemichal data & & \\
Total cholesterol (mg/dl) & $182 \pm 37$ & $165 \pm 27$ \\
LDL cholesterol (mg/dl) & $103 \pm 36$ & $99 \pm 28$ \\
HDL cholesterol (mg/dl) & $50 \pm 12$ & $49 \pm 8$ \\
Triglycerides (mg/dl) & $123 \pm 50$ & $144 \pm 59$ \\
Medical history and medication & 8 \\
Previous ACS & 0 & 17 \\
Statins & 0 & \\
\hline
\end{tabular}

NSTEACS non-ST elevated ACS, SD standard deviation 


\subsubsection{Sample preparation}

To perform the metabolomic analysis of plasma samples by GC/MS, we employed the protocol described by Jiye et al. with minor modifications (Jiye et al. 2005). Briefly, $50 \mu \mathrm{l}$ of thawed plasma was vortex-mixed with $50 \mu$ of acetonitrile to precipitate the plasma proteins and centrifuged at $12,000 \times g$ for $5 \mathrm{~min}$. $75 \mu \mathrm{l}$ of supernatant was carefully separated and dried out in a heating block at $70{ }^{\circ} \mathrm{C}$ (Thermomixer comfort, Eppendorf) for $1 \mathrm{~h}$. This dried aliquot was derivatized by methoximation adding $40 \mu \mathrm{l}$ methoxiamine hydrochloride $(20 \mathrm{mg} / \mathrm{dL})$ in pyridine and kept for $24 \mathrm{~h}$ at room temperature. Later, $10 \mu \mathrm{l}$ of BSTFA $+1 \%$ TMCS was added and kept for $1 \mathrm{~h}$ in a heating block at $70{ }^{\circ} \mathrm{C}$ for $60 \mathrm{~min}$ for trimethylsilylation (Fig. 1a). Finally, $5 \mu \mathrm{l}$ of methyl pentadecanoate (IS) was added and the mixture was centrifuged at $12,000 \times g$ for $5 \mathrm{~min}$. Supernatant $(50 \mu \mathrm{l})$ was separated for GC-MS analysis.

\subsubsection{Experimental conditions}

The GC-MS system consisted of an Agilent 6890N GC system, coupled to an Agilent 5975c single quadrupole MSD and a capillary column HP-5MS $5 \%$ Siloxane Phenyl

\section{A Plasma metabolomic profiling}
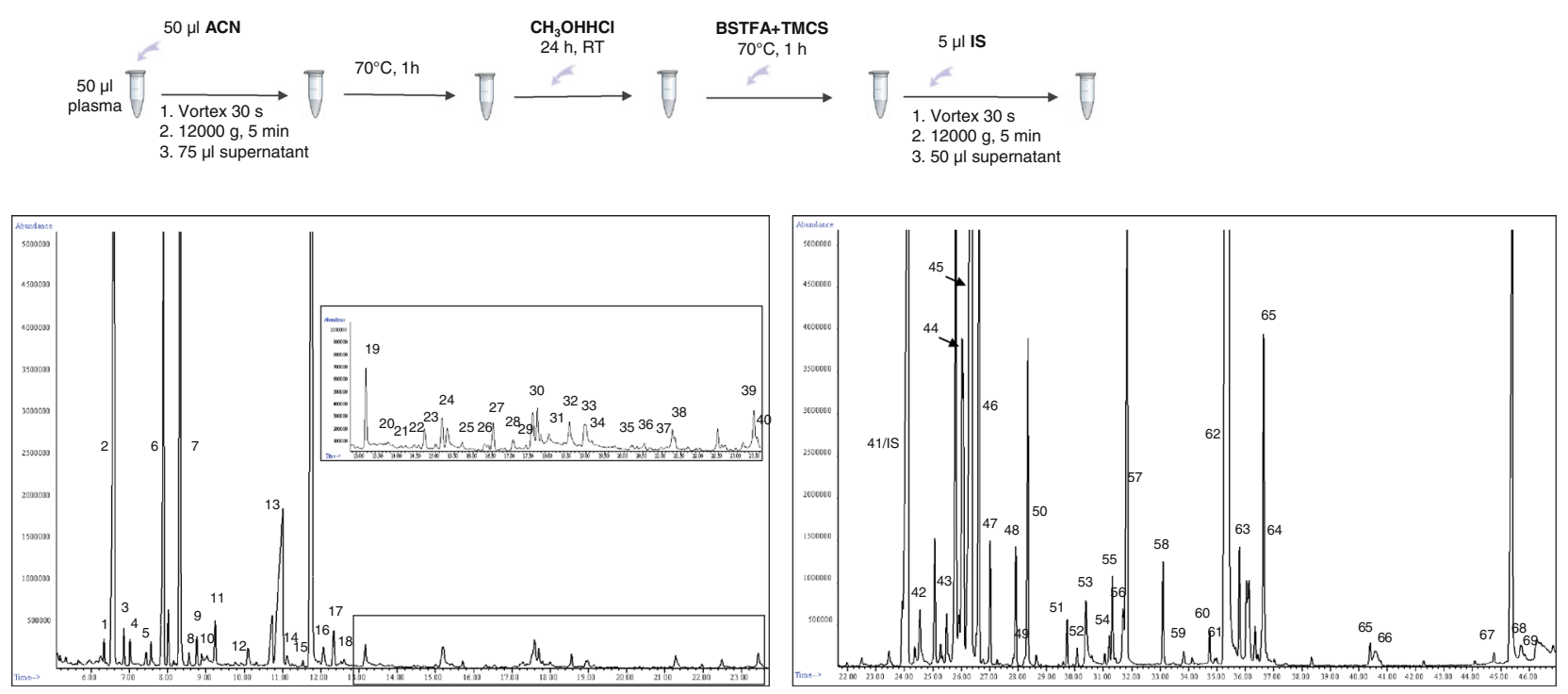

\section{B Fatty acids profiling}
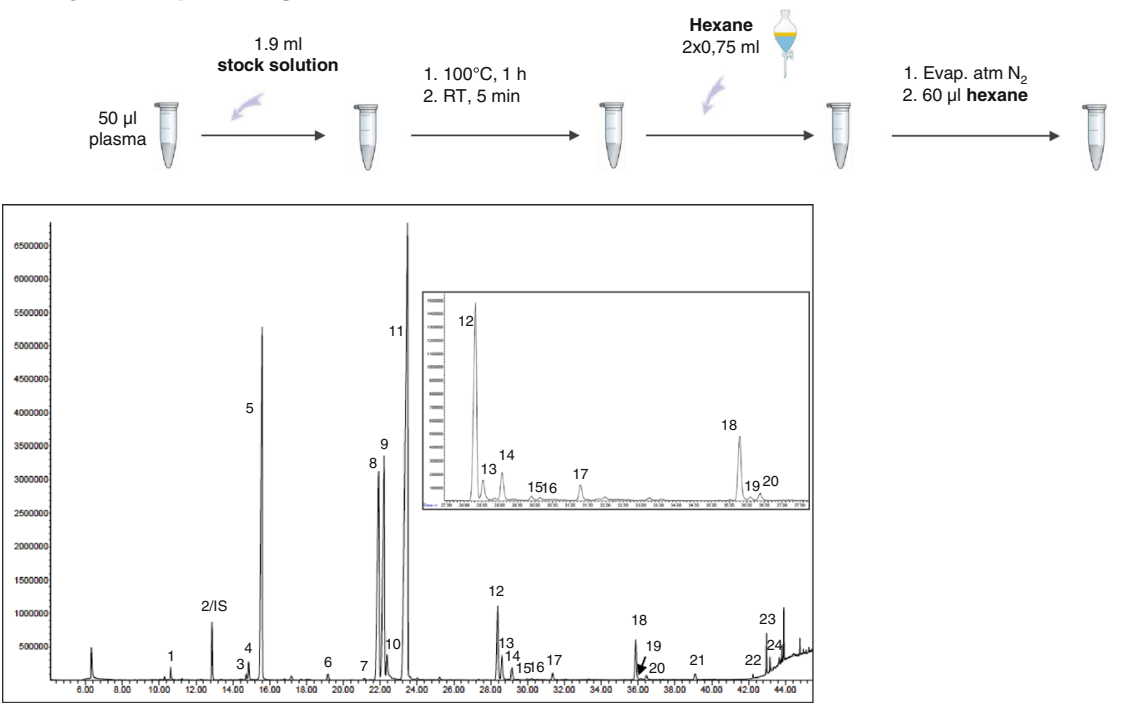

Fig. 1 Protocols for metabolomic analysis of plasma profiling (a) and fatty acids profiling (b) with a representative chromatogram including the identified metabolites summarized in Table $2 \mathrm{a}$, c respectively 
Methyl $(30 \mathrm{~m} \times 250 \mu \mathrm{m} \times 0.25 \mu \mathrm{m})$ (Agilent Technologies, USA). Derivatized samples $(1 \mu \mathrm{l})$ were injected into the GC-MS system in splitless mode. It is important to point out that all samples were injected in triplicate and the average normalized peak area for every metabolite was used for further analysis. The GC oven was programmed with an initial temperature of $60{ }^{\circ} \mathrm{C}$ and then increased to $285{ }^{\circ} \mathrm{C}$ at $5{ }^{\circ} \mathrm{C} / \mathrm{min}$ and held at the final temperature for $2 \mathrm{~min}$. Highpurity helium was used for the carrier gas at a flow rate of $1.5 \mathrm{~mL} / \mathrm{min}$. The injection port temperature was $230{ }^{\circ} \mathrm{C}$ and the interface temperature was set at $290{ }^{\circ} \mathrm{C}$. The EI source temperature was set at $230{ }^{\circ} \mathrm{C}$ with energy of $70 \mathrm{eV}$. The selected mass range was $50-600 \mathrm{~m} / \mathrm{z}$ and the selected scan speed was 0.99 scans per second. Identification of GC-MS detected peaks was performed by direct comparison of their mass spectral with the NIST02 MS library.

\subsection{Fatty acids profiling}

\subsubsection{Chemicals and reagents}

All reagents used for the metabolomic analysis of plasma fatty acid; acetyl chloride, methyl pentadecanoate, methanol and hexane were purchased from Sigma-Aldrich. The ultrapure milli Q water was produced by a Milli-Q Reagent Water System (Millipore).

\subsubsection{Transesterification reaction}

The transesterification reaction carried out for the metabolomic analysis of plasma fatty acid was performed following the method described by Lepage and Roy in (1986) with minor modifications. For every work session, a stock solution of new transesterification reagents was prepared. This stock solution contained $1.7 \mathrm{ml}$ of methanol, $100 \mu \mathrm{l}$ of acetyl chloride and $100 \mu \mathrm{l}$ of a $100 \mathrm{ppm}$ solution of methyl pentadecanoate (IS). Briefly, $50 \mu \mathrm{l}$ of plasma were combined with $1.9 \mathrm{ml}$ of stock solution in an eppendorf tube, which was heated at $100{ }^{\circ} \mathrm{C}$ for $1 \mathrm{~h}$ in a heating block (Thermomixer comfort, Eppendorf). Afterwards, it was allowed to cool to room temperature for $5 \mathrm{~min}$ and $0.75 \mathrm{ml}$ of hexane was added and vigorously vortex for $30 \mathrm{~s}$. This extraction process was repeated twice. After every extraction, supernatant was collected using a Pasteur pipette and the extracts were combined into a new eppendorf tube, which was taken to dryness under nitrogen. After redissolving in hexane $(60 \mu \mathrm{l})$ the dry residue the sample was ready for GC-MS analysis (Fig. 1b).

\subsubsection{Experimental conditions}

The temperature program used in a chromatographic analysis depends on the nature of the metabolites to be separated. In this case, the GC oven was programmed with an initial temperature of $130{ }^{\circ} \mathrm{C}$ for $1 \mathrm{~min}$, increased to $178{ }^{\circ} \mathrm{C}$ at $4^{\circ} \mathrm{C} / \mathrm{min}$, then increased to $225^{\circ} \mathrm{C}$ at $1{ }^{\circ} \mathrm{C} / \mathrm{min}$ and finally an increasing of $40{ }^{\circ} \mathrm{C} / \mathrm{min}$ to $245^{\circ} \mathrm{C}$. This final temperature was held for 13 min completing a chromatographic cycle of $45 \mathrm{~min}$. The injection of the sample $(1 \mu \mathrm{l})$ was performed in split mode with a split ratio of 7.5:1. It is important to note that all samples were injected in triplicate and the average normalized peak area for every metabolite was used for further statistical analysis. Helium was used as carrier gas at a flow of $2.7 \mathrm{ml} / \mathrm{min}$. The injector temperature and interface were 230 and $300{ }^{\circ} \mathrm{C}$ respectively. The EI source temperature was set at $230{ }^{\circ} \mathrm{C}$ with energy of $70 \mathrm{eV}$. Finally, the acquisition was performed with a SCAN-mode in a mass range $50-600 \mathrm{~m} / \mathrm{z}$ and a scan ratio of 0.99 scans per second.

\subsection{Identification of metabolites}

The analysis and integration of every obtained chromatogram from each injected sample was performed using the ChemStation software (Agilent Technologies). The identification of different metabolites was carried out by direct comparison of experimental mass spectra with the theoretical mass spectra contained in the 2.0 NIST library (National Institute of Standards and Technology).

Data acquisition was performed in SCAN mode, which consists of monitoring abroad range of mass fragments (e.g. 50-500 U) in order to identify all known compounds present in the sample. The mass spectra of all chromatographic peaks were compared with the mass spectra contained in the NIST 2.0 library. Every peak area were then normalized using the IS (methyl pentadecanoate) peak area in order to find metabolites that were altered between the experimental groups. For the statistical and multivariate analysis the Microsoft Excel add-in software XLSTAT 2013 and SPSS v15.0 were used.

\subsection{Validation}

\subsubsection{GC/MS}

For the validation process by GC/MS, the same chemicals, sample preparation protocol and experimental conditions previously explained in Plasma metabolomic profiling (2.2) were used.

\subsubsection{LC-MS/MS}

2.5.2.1 Sample preparation Protein removal from plasma samples was performed by precipitation in $50 \%$ acetonitrile. Following centrifugation, supernatant was taken for metabolite analysis, filtered through $0.20 \mu \mathrm{m}$ and 
mixed 1:1 with mobile phase $\mathrm{A}(0.1 \%$ formic acid in double distilled water Milli-Q Millipore System) for LC(QQQ) MS analysis in SRM mode.

2.5.2.2 Experimental conditions The HPLC system consisted of a degasser, two binary pumps and a thermostated autosampler maintained at $4{ }^{\circ} \mathrm{C}$ (1200 Series, Agilent Technologies). A sample volume of $5 \mu \mathrm{l}$ was injected in a reversed-phase column $(1.8 \mu \mathrm{m}, 2.1 \times 50 \mathrm{~mm}$ Zorbax, Agilent Technologies) thermostated at $25^{\circ} \mathrm{C}$. Separation took place at $0.5 \mathrm{ml} / \mathrm{min}$ in an acetonitrile gradient: 3-95 \% B at $9 \mathrm{~min}, 95 \% \mathrm{~B}$ for $1 \mathrm{~min}$ (phase B: $0.1 \%$ formic acid in acetonitrile). A 6460 Triple Quadrupole Mass Spectrometer (Agilent Technologies) was on-line coupled to the HPLC system and controlled by Mass Hunter Software (v4.0). The mass spectrometer was operated in positive ion mode with $150{ }^{\circ} \mathrm{C}$ source gas temperature and $11 \mathrm{l} / \mathrm{min}$ source gas flow. Fragmentor was optimized for each metabolite in the range 130-175 V, dwell time was fixed to $200 \mathrm{~ms}$ and so delta EMV to 200 V. Optimal SRM transitions were selected in direct infusion mode by previous analysis of commercial metabolite standards, injected separately. Collision energy was optimized for each metabolite by means of Optimizer Software (Agilent Technologies). Measured transitions were: 2-OH-butyric acid $(105.1>77.1), \quad \mathrm{CE}=18 \mathrm{~V}$; 3-OH-butyric acid (105.1 > 45.2), $\mathrm{CE}=14 \mathrm{~V}$ and 5-OHtryptophan $(220.1>133.0), \mathrm{CE}=34 \mathrm{~V}$. Individual signals were normalized based on TIC (total ion current) to account for sample dilution variability and normalized peak areas for comparison were calculated.

\section{Results}

\subsection{Plasma metabolomic profiling}

A total of 68 metabolites (not considering IS) were identified in healthy subjects and NSTACS patients. In both groups, the relative standard deviations (\% RSD) for every peak areas were calculated. Results are shown in Table $2 \mathrm{a}$ with the name of identified metabolites, their retention times (RT) and their position in the chromatogram.

Principal component analysis (PCA) was used as multivariate non-targeted analysis. Two well-separated groups were obtained in the score plot (Fig. 2a) suggesting that the plasma metabolomic profiling of the NSTACS patients was significantly different from that of the healthy subjects.

Statistical analysis using a Student's $t$-test of the normalized areas of every chromatographic peak resulted in the existence of 18 metabolites (alanine, 2-OH-butyric acid, 3-OH-butyric acid, 2-keto-3-methylvaleric acid, serine, leucine, threonine, glycine, 2-OH-benzoic acid, 5-OH- tryptophan, palmitic acid, linoleic acid, cis-vaccenic acid, lactate, alpha-OH-valeric acid, citrate, 5-oxo-proline and stearic acid) whose normalized peak areas significantly varied $(p<0.05)$ between both experimental groups (Table 2b). Of these, 10 metabolites (2-OH-butyric acid, 3-OH-butyric acid, 2-OH-benzoic acid, palmitic acid, citrate, cis-vaccenic acid, lactate, alpha-OH-valeric acid, 5-oxo-proline and stearic acid) were elevated in NSTEACS patients and 8 metabolites (alanine, 2-keto-3-methylvaleric acid, serine, leucine, threonine, glycine, 5-OH-tryptophan and linoleic acid) were significantly decreased.

\subsection{Fatty acids profiling}

In our metabolomic study of plasma fatty acids, the method described by Lepage and Roy in (1986) allowed us to exclusively extract the fatty acid fraction from plasma. This extraction protocol permitted to identify 21 new fatty acids with a very low concentration whose peaks were masked by more abundant metabolites in the plasma metabolomic profiling in which 68 metabolites were identified.

A total of 24 fatty acids were identified by GC-MS. Figure $1 \mathrm{~b}$ shows a representative chromatogram where peak numbers match to the metabolites listed in Table 2c.

PCA showed two well-separated groups in the score plot suggesting a particular and characteristic fatty acid profiling for NSTEACS patients (Fig. 2b). The statistical analysis using a Student's $t$-test revealed four fatty acids [palmitic acid (16:0), trans-palmitoleic (16:1n-9), cis-vaccenic acid (18:1n-7) and linoleic acid (18:2n-6)] whose concentrations were statistically altered $(p<0.05)$ between both experimental groups. Of these four metabolites, three fatty acids (palmitic acid, trans-palmitoleic, and cis-vaccenic acid) were elevated in NSTEACS patients while the linoleic acid was decreased in this group. Furthermore, three of four significantly altered fatty acids in this study (palmitic acid, cis-vaccenic acid and linoleic acid) were also significantly altered in the plasma metabolomic study while the stearic acid in this analysis showed no statistically significant difference.

\subsection{Validation of results by GC/MS}

Despite a preceding set of samples had been already analyzed by GC/MS, the particular and tedious conditions for sample derivatization encouraged us to confirm the validity of the results previously obtained. For this purpose, a new and independent group of 15 NSTEACS patients and 15 healthy controls was used.

A Student's $t$-test was performed using the normalized areas of the 18 metabolites, which have previously showed statistical significance $(p<0.05)$. In this case, 14 metabolites (2-OH-butyric acid, 3-OH-butyric acid, 2-keto-3- 
Table 2 Summary of all identified metabolites in plasma metabolomic profiling (Table 2a), fatty acids profiling (Table 2c)

\begin{tabular}{|c|c|c|c|c|c|c|}
\hline \multicolumn{7}{|l|}{ A } \\
\hline Metabolite & R.T. (min) & Peak & \multicolumn{2}{|l|}{ Metabolite } & R.T. (min) & Peak \\
\hline Piruvate & 6.241 & (1) & \multicolumn{2}{|c|}{ 2,3,4,5-Tetrahydroxypentanoic acid-1,4-lactone } & 20.343 & $(36)$ \\
\hline Lactate & 6.516 & $(2)$ & \multicolumn{2}{|l|}{ Trihydroxypentanoic acid } & 21.132 & $(37)$ \\
\hline Glycolate & 6.778 & (3) & \multicolumn{2}{|l|}{ 5-OH-tryptophane } & 21.292 & $(38)$ \\
\hline Valine & 6.947 & (4) & \multicolumn{2}{|l|}{ Glycerate 3P } & 23.459 & $(39)$ \\
\hline Alanine & 7.371 & $(5)$ & \multicolumn{2}{|l|}{ Ribonic acid } & 23.530 & $(40)$ \\
\hline Alpha-hydroxyisobutyric acid & 7.969 & (6) & \multicolumn{2}{|l|}{ Methyl pentadecanoate (IS) } & 24.129 & $(41)$ \\
\hline Oxalic acid & 8.287 & (7) & \multicolumn{2}{|l|}{ Isocitric acid + Tetradecanoic acid } & 24.533 & $(42)$ \\
\hline Leucine $(\mathrm{COOH})$ & 8.531 & $(8)$ & \multicolumn{2}{|l|}{ D-Fructose } & 25.832 & (43) \\
\hline Beta-hydroxybutyric acid & 8.734 & (9) & \multicolumn{2}{|l|}{ D-Glucose } & 26.349 & $(44)$ \\
\hline Alpha-hydroxyvaleric acid & 8.859 & $(10)$ & \multicolumn{2}{|l|}{ D-Galactose } & 26.617 & $(45)$ \\
\hline Isoleucine $(\mathrm{COOH})$ & 9.009 & $(11)$ & \multicolumn{2}{|l|}{$\mathrm{N}$-acetil glucosamine } & 26.761 & $(46)$ \\
\hline 2-keto-3-methylvaleric acid & 9.753 & (12) & \multicolumn{2}{|l|}{ Glucitol } & 27.014 & $(47)$ \\
\hline Urea & 10.990 & (13) & \multicolumn{2}{|l|}{ Beta-D-glucopiranose } & 27.913 & $(48)$ \\
\hline Serine $(\mathrm{COOH})$ & 11.136 & (14) & \multicolumn{2}{|l|}{ Gluconic acid } & 28.214 & (49) \\
\hline Leucine (COO-SiMe3) & 11.537 & $(15)$ & \multicolumn{2}{|l|}{ Palmitic acid } & 28.353 & $(50)$ \\
\hline Threonine $(\mathrm{COOH})$ & 12.101 & (16) & \multicolumn{2}{|l|}{ Inositol } & 29.532 & $(51)$ \\
\hline Glycine & 12.340 & (17) & \multicolumn{2}{|l|}{ Heptadecanoic acid } & 30.089 & $(52)$ \\
\hline Succinate & 12.525 & $(18)$ & \multicolumn{2}{|l|}{ 6-Hydroxy- $\alpha$-methylnaphthaleneacetic acid } & 30.386 & $(53)$ \\
\hline Glycerate & 13.166 & (19) & \multicolumn{2}{|l|}{ Linoleic acid } & 31.228 & $(54)$ \\
\hline$(\mathrm{R}, \mathrm{S})$-2,3-Dhydroxybutanoic acid & 13.766 & $(20)$ & \multicolumn{2}{|l|}{ Oleic acid } & 31.342 & $(55)$ \\
\hline Serine (O-SiMe3) & 13.869 & $(21)$ & 11-Cis-octadecenoic acid & & 31.441 & $(56)$ \\
\hline Threonine (O-SiMe3) & 14.577 & $(22)$ & Estearic acid & & 31.834 & $(57)$ \\
\hline Aspartic acid (COOH) & 14.739 & (23) & Acenaphthylene & & 33.110 & $(58)$ \\
\hline Homocysteine & 15.240 & (24) & $9,12,15$-Octadecatrienoic acid glycerol & & 33.815 & $(59)$ \\
\hline (R,S)-3,4-dihydroxybutanoic acid & 15.714 & $(25)$ & Terbutaline & & 34.756 & $(60)$ \\
\hline Aspartic acid (O-SiMe3) & 16.321 & (26) & Araquidic acid & & 35.000 & $(61)$ \\
\hline Aminomalonic acid & 16.547 & $(27)$ & EDTA & & 35.408 & $(62)$ \\
\hline Malic acid & 17.083 & (28) & 6-Octadecanoate- $\alpha$-D-Glucopyranoside & & 35.775 & $(63)$ \\
\hline 2-Hydroxybenzoic acid & 17.292 & (29) & Ribofuranose & & 36.651 & $(64)$ \\
\hline 5-Oxo-proline & 17.602 & $(30)$ & Disaccharide glucose derivate & & 40.411 & $(65)$ \\
\hline Phenylalanine & 18.019 & $(31)$ & D-Turanose & & 40.773 & $(66)$ \\
\hline Threonic acid & 18.564 & $(32)$ & Cholesterol & & 44.777 & $(67)$ \\
\hline Erytronic acid & 18.995 & $(33)$ & $9,12,15$-Octadecatrienoic acid glycerol & & 45.705 & $(68)$ \\
\hline Cystathionine & 19.169 & (34) & 1-Monolinoleoylglycerol & & 45.846 & $(69)$ \\
\hline 4-Hydroxyphenylacetic acid & 20.225 & $(35)$ & & & & \\
\hline B & & & & & & \\
\hline Decreased metabolites & $t$ & Sig. (bilateral) & Increased metabolites & $t$ & Sig. & ateral) \\
\hline Alanine & 5.890 & $<0.001$ & 2-OH-benzoic acid & -11.570 & $<0$. & \\
\hline Threonine & 5.727 & $<0.001$ & 2-OH-butyric acid & -7.056 & $<0$. & \\
\hline Serine & 5.163 & $<0.001$ & Citrate & -5.667 & $<0$. & \\
\hline Glycine & 5.040 & $<0.001$ & 3-OH-butyric acid & -4.968 & $<0$. & \\
\hline 5-OH-tryptophan & 4.057 & 0.001 & Palmitic acid & -3.660 & 0.0 & \\
\hline 2-Keto-3-methylvaleric acid & 3.805 & 0.002 & Cis-vaccenic acid & -3.140 & 0.0 & \\
\hline Leucine & 2.719 & 0.014 & Alpha-OH-valeric acid & -2.868 & 0.0 & \\
\hline Linoleic acid & 2.708 & 0.014 & Stearic acid & -2.809 & 0.0 & \\
\hline & & & Lactate & -2.623 & $0 .($ & \\
\hline
\end{tabular}


Table 2 continued

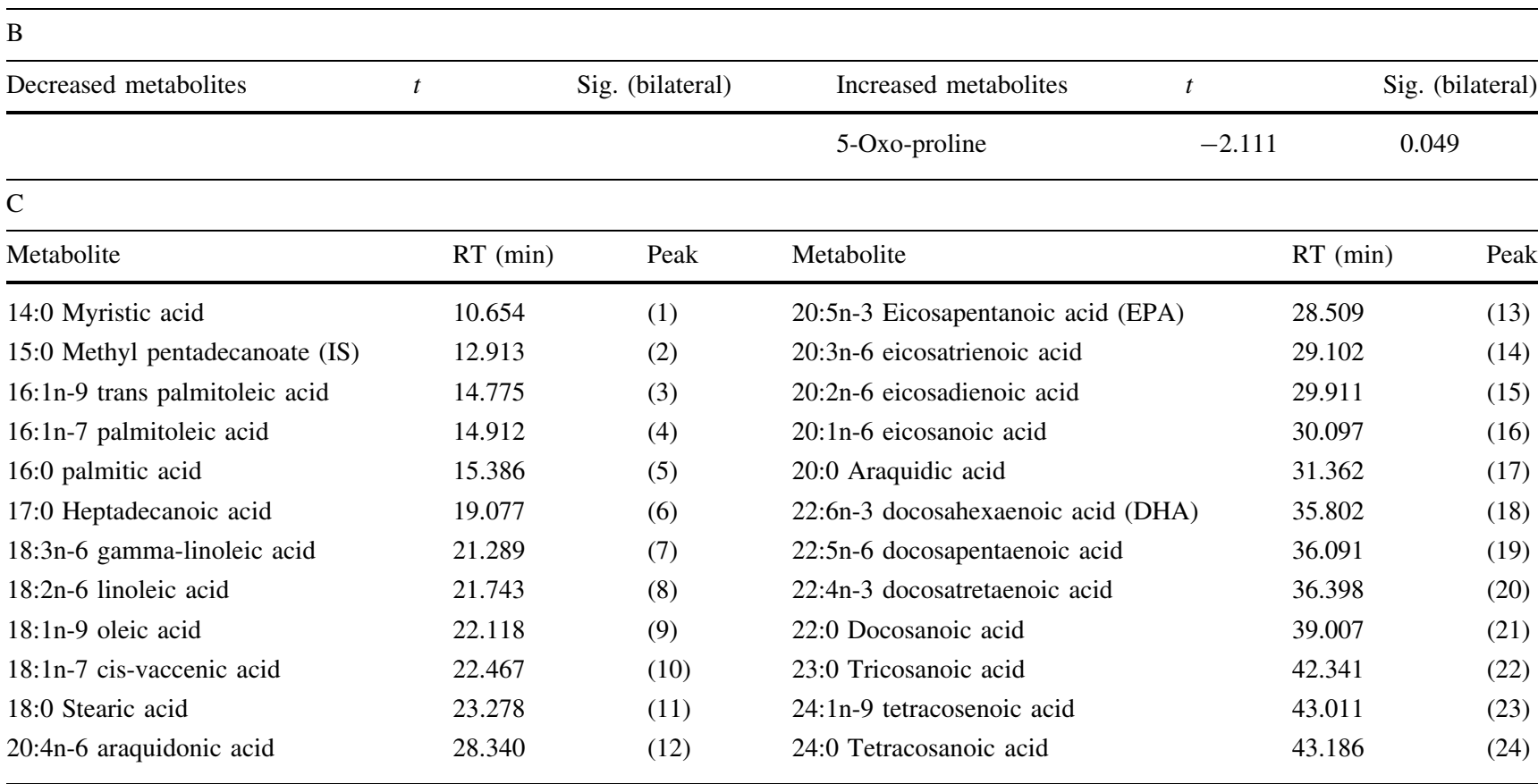

Student's $t$-test analysis revealed the existence of eifgt significantly decreased metabolites and ten significantly increased metabolites in NSTEACS patients. In the case of amino acids normalized areas of the free [e.g., Serine $(\mathrm{COOH})$ ] and derivatized fractions [e.g., Serine (OSiMe3)] were added for statistical analysis (Table 2b)

Fig. 2 Results of PCA for plasma metabolomic analysis (a) and plasma fatty acid analysis (b). In the score plot, healthy controls (left, blue) and NSTEACS patients (right, red) are represented (Color figure online)
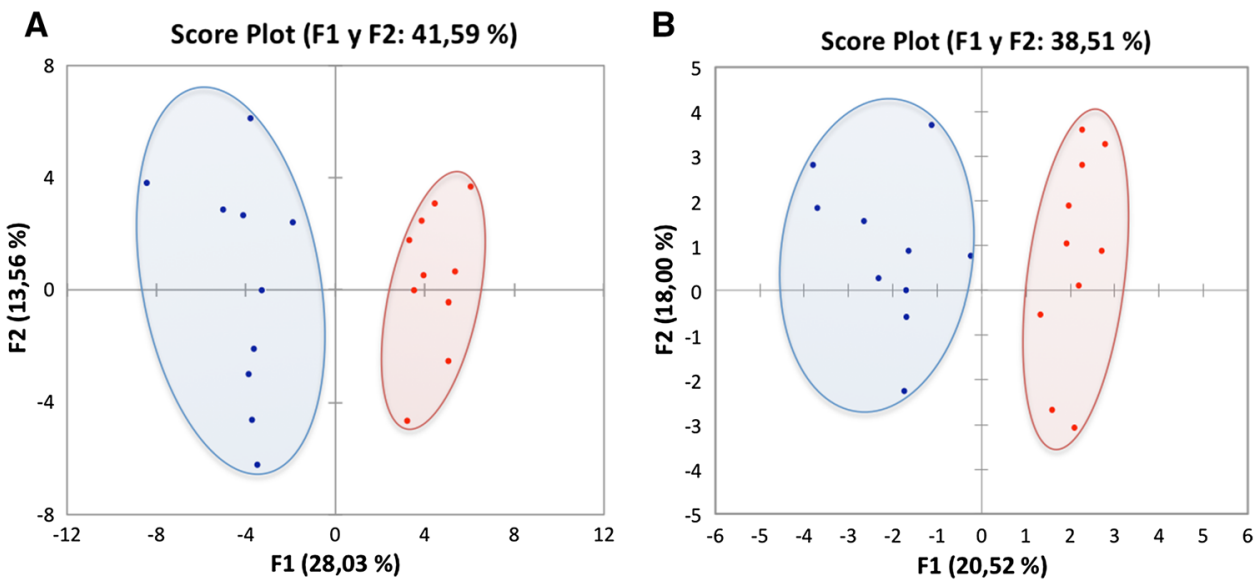

methylvaleric acid, serine, leucine, alanine, threonine, glycine, 2-OH-benzoic acid, 5-OH-tryptophan, citrate, palmitic acid, linoleic acid and cis-vaccenic acid) showed statistically significant differences. However, four metabolites (lactate, alpha-OH-valeric, 5-oxo-proline and stearic acid) did not show statistical significance $(p<0.05)$ (Fig. 3).

Additionally, it was interesting to find out that the three fatty acids, which were also found altered in both, plasma profiling and fatty acids analysis (palmitic acid, cis-vaccenic acid and linoleic acid), were validated.

Between the 14 metabolites that showed statistically significant differences $(p<0.05)$ in the validation process, the 2-OH-benzoic acid or salicylic acid was not considered as a potential biomarker for CVD since it is the major metabolite in the catabolism of 2-(acetyloxy)-benzoic or acetylsalicylic acid (aspirin) which constitutes a cornerstone in the initial therapy for ACS.

\subsection{Validation of results by LC/MS}

Complementary, a new metabolic analysis by liquid chromatography coupled with tandem mass spectrometry (LCMS/MS) was also performed on a different set of samples 
A

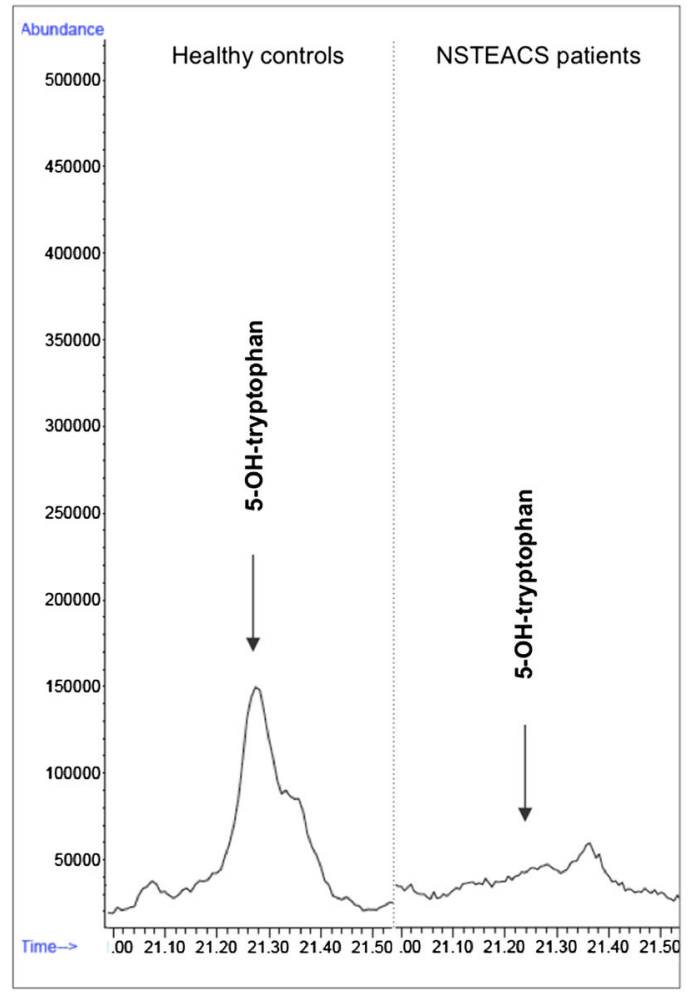

B

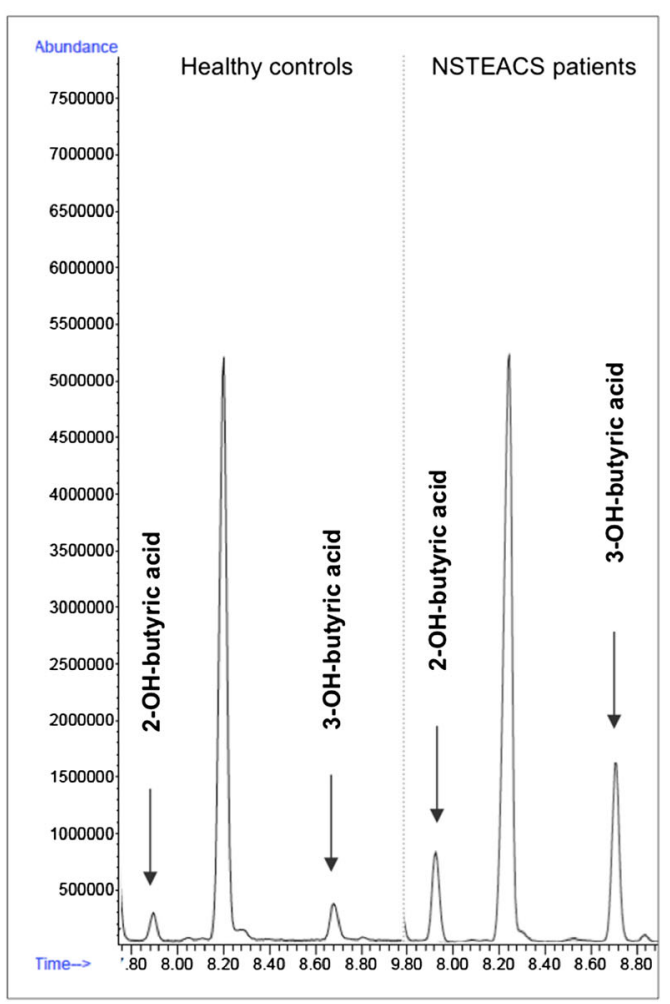

Fig. 3 5-OH-tryptophan (21.292 min) showed decreased levels in NSTEACS patients compared to healthy controls (a). 2-OH-butyric acid (7.969 $\mathrm{min})$ and 3-OH-butyric acid $(8.734 \mathrm{~min})$ showed increased plasma levels in NSTEACS patients compared to healthy controls $(\mathbf{b})$

including 10 NSTEACS patients and 10 healthy controls using a triple quadrupole 6460 series mass spectrometer (Agilent Technologies) and the selected reaction monitoring (SRM) technique.

Using SRM, three metabolites, 5-OH-tryptophan, 2-OHbutyric acid and 3-OH-butyric acid showed statistical significance $(p<0.05)$ (Fig. 4).

\section{Discussion}

The diagnosis of ACS and the assignment to a risk group should be undertaken as rapidly as possible. Unfortunately, the high risk of suffering an ACS cannot be identified using the existing clinical biomarkers and when the first symptoms appear, the disease is already in an irreversible state. A great number of novel potential biomarkers have been explored in recent years to discover their usefulness in clinical practice for early diagnosis and correct individual risk stratification (Baldus et al. 2003; Brennan et al. 2003; Heeschen et al. 2003; Varo et al. 2003). According to the multifactorial nature of atherosclerosis, the combined use of biomarkers for myocardial necrosis, inflammation and myocardial and renal dysfunction have evidenced $o$ improve risk stratification (James et al. 2003; Heeschen et al. 2000).
For metabolomic-based studies, GC/MS offers high sensitivity, high reproducibility and optimal chromatographic resolution, which make it an excellent tool for identification of biological or pathological variations that occurs in human diseases. These characteristics are particularly important when analyzing the complex mixtures of metabolites found in biofluids, such as serum/plasma and urine, or even in tissue extracts. Many studies have been published to date for identification of novel potential biomarkers in urine (Paige et al. 2007; Chen et al. 2008) and plasma (Meyer et al. 2011; Ooi et al. 2011; Han et al. 2011; Zeng et al. 2010; Vallejo et al. 2009), which proves that GC/MS and other metabolomic approaches offer promising results for the elucidation of disease mechanisms and biomarker discovery in biofluids.

\subsection{Defining a candidate panel of biomarkers}

The results of our metabolic analysis of plasma in NSTEACS patients versus healthy controls reveal the complex network of altered metabolic pathways in the course of ACS. We considered that hypoxia constitutes the main axis to understand and explain how the metabolism of every cell must adapt to the deficiency of oxygen in order to maintain their metabolic activity. These changes produce several alterations in the normal functioning of many metabolic 
A

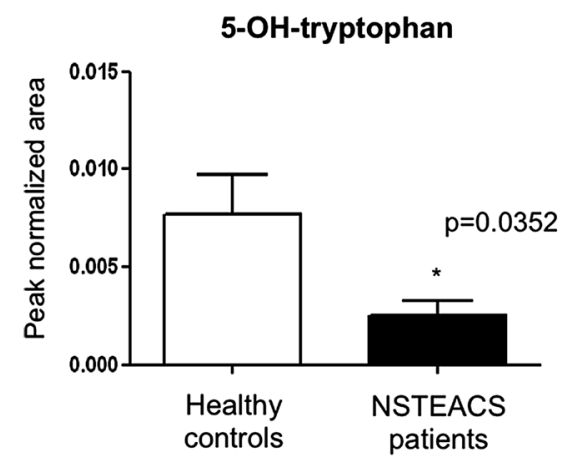

B

5-OH-tryptophan

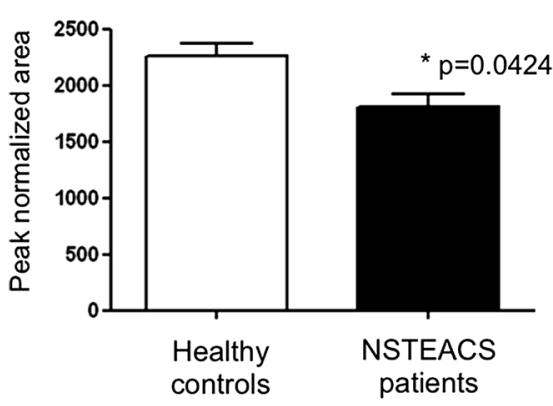

2-OH-butyric acid

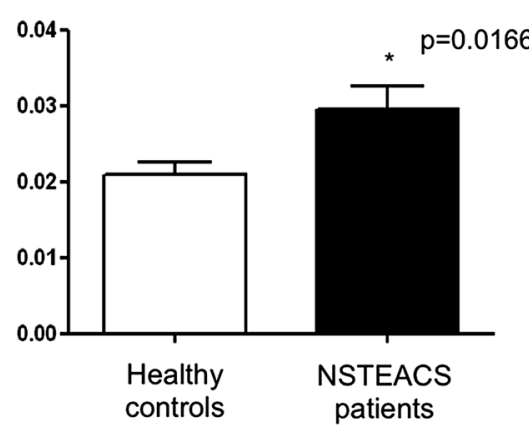

2-OH-butyric acid

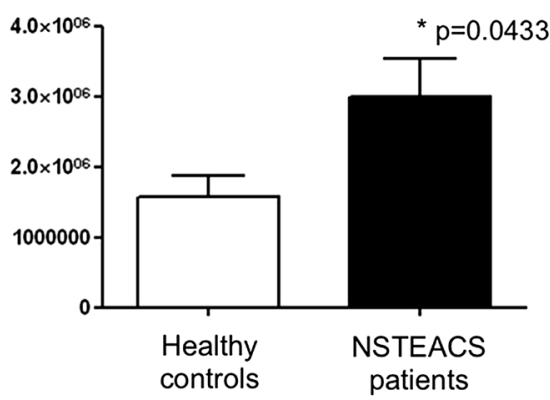

3-OH-butyric acid

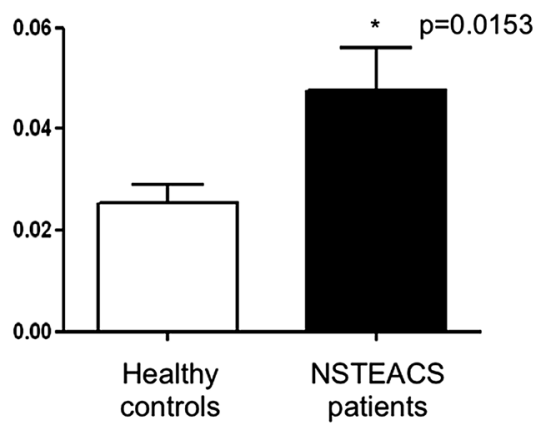

3-OH-butyric acid

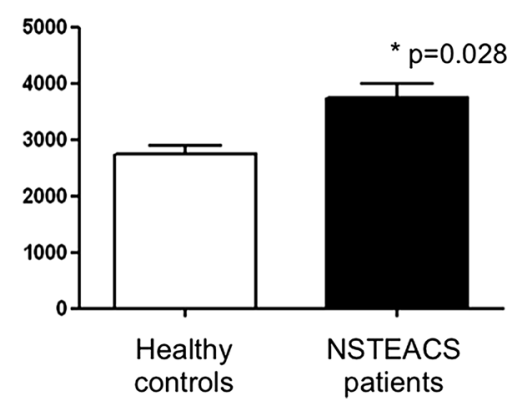

Fig. 4 Validation results by GC/MS (a) and SRM (b). The three metabolites showed statistically significant changes between the two experimental groups

pathways that probably interact each other trying to recover the equilibrium state. The alterations we found in three special metabolites, 5-OH-tryptophan, 2-OH-butyric acid and 3-OH-butyric acid allow a better understanding of the changes that occur in heart cells.

\subsubsection{5-OH-tryptophan}

The existence of quantitative alterations in plasma levels of amino acids and their metabolites can be explained considering either a decrease in anabolic activity of these metabolites or an increase in the catabolic pathways. These have been also previously described in several CVDs with diverse results (Lewis et al. 2008; Mayr et al. 2008).

The 5-OH-tryptophan is the first metabolite of tryptophan in the biochemical pathway for serotonin synthesis. We suggest that under hypoxia conditions, the diminution of plasmatic levels of 5-OH-tryptophan may reflect a diminished synthesis of serotonin since cells prioritize the use of tryptophan as a precursor of glucose versus serotonin production.

\subsubsection{2-OH-butyric acid}

Glutathione is the main endogenous antioxidant produced by cells. It neutralizes free radicals and reactive oxygen compounds, as well as maintaining exogenous antioxidants like vitamins $\mathrm{C}$ and $\mathrm{E}$ in their reduced forms. Oxidative stress conditions dramatically increase the rate of hepatic synthesis of glutathione (García-Pinilla et al. 2008). It is synthesized from the amino acids cysteine, glutamic acid and glycine. Under these conditions of metabolic stress, the supply of cysteine for glutathione synthesis become limiting and homocysteine is used to form cystathionine, which is cleaved to cysteine and 2-OH-butyric acid. Thus elevated plasma levels of 2-OH-butyric acid can be a good biomarker of cellular oxidative stress for the early diagnosis of ACS.

\subsubsection{3-OH-butyric acid}

Another altered metabolite of similar structure was 3-OHbutyric acid, a ketone body together with the acetoacetate, and acetone. Elevated levels of ketone bodies in blood and urine mainly occur in diabetic ketoacidosis (Arora and Menchine 2012). Type 1 diabetes mellitus (DMI) patients have decreased levels of insulin in the blood that prevent glucose enter cells so these cells use the catabolism of fats as energy source that produce ketones as final products. We hypothesize that the high plasma levels observed in NSTEACS patients can be due to hypoxia more than to DMI, since just $16 \%$ of our NSTEACS patients had been 
diagnosed with DMI. However, pre-diabetic conditions in patients such as impaired glucose tolerance and insulin resistance should also be taken into account in terms of 3-OH-butyric acid blood levels. In this theoretical scenario, we postulate that the hypoxia situation comes to "mimic" the physiological situation that occurs in DMI. In this case, the low energy yield of glucose metabolism "forces" these cells to use fat as energy source (through catabolism independent of aerobic/anaerobic conditions) occurring ketones as final products. In our experiment, the 3-OHbutyric acid was strongly elevated in NSTEACS patients compared to healthy controls which would reflect the situation of hypoxia that are suffering the heart cells.

Finally, this exciting theoretical basis is supported by our experimental results obtained through two different chromatographic techniques (GC-MS and LC-MS). Despite these interesting results, further studies will be necessary to achieve a stronger evidence of its validity. If these confirm the prior results presented in this study, we strongly believe that the panel of biomarkers consisting of 5-OH-tryptophan, 2-OH-butyric acid and 3-OH-butyric acid will have a great potential for early diagnosis of ACS.

\subsection{Drawbacks of the study}

In this study, our goal has been to establish a preliminary vision of characteristic metabolomic profiles of ACS patients compared with healthy controls. According to this initial approach, these results have been explained in the context of available clinical and biochemical data with no significant restrictions on diet applied to patients or healthy individuals.

Many of the well-known cardiovascular risk factors may influence the metabolomic profile. Additionally, in the case of NSTEACS patients, the previous treatment with statins could generate characteristic peak in the profile although no metabolites derived from the statins were found in this study.

Among the cardiovascular risk factors, smoking remains one of the most important. It is extensively documented that smoking has several effect in the body. Primarily, it affects to the respiratory and circulatory systems reducing the lung function and breathlessness due to swelling and narrowing of the lung airways, decreasing the oxygen carried by the blood, raising blood pressure and heart rate and sticking the blood increasing the risk of stroke and heart attack between many others. Smoking also affects to immune system increasing the risk of lung infections and reduces fertility in both, males and females. In this study, smokers represent the $28 \%$ in the NSTACS patients group and the $8 \%$ in the healthy controls group. In this study, first principal component clearly separates both experimental groups and therefore we considered that, in this case, smoking showed a minor influence in comparison to the pathology. However, according to these several effects, and in order of clearly elucidate the influence of smoking on the metabolite profile, a separately study must be performed using a particular group of smokers NSTACS patients.

\subsection{Future directions}

Globally, the conclusions reached in this study should be confirmed with further studies to be performed in a more rigorous selection of patients. It is evident that every cardiovascular risk factor (smoking, diabetes, high LDLcholesterol, etc.) could individually affect the plasma metabolic profile and therefore this influence must be independently studied. New metabolomic studies with selected groups of patients will allow us to check the validity of these results and to clarify the influence of every singular risk factor in the altered metabolites.

Acknowledgments This work was supported by grants from the Instituto de Salud Carlos III (FIS PI070537, FIS PI080970, FIS PI080920, RD07/0064/0023, PI11/02239, PI11/001401, CP09/ 00229), Fondo de Investigación Sanitaria de Castilla la Mancha (FISCAM, PI2008/08; FISCAM PI2008/52) and Redes Temáticas de Investigación Cooperativa (FONDOS FEDER, RD06/0014/1015, RD12/0042/0071). We thank the staff of laboratory of biochemistry (Hospital Virgen de la Salud) for their contribution and technical support.

\section{References}

Arora, S., \& Menchine, M. (2012). The role of point-of-care $\beta$ hydroxybutyrate testing in the diagnosis of diabetic ketoacidosis: A review. Hospital Practice (1995), 40(2), 73-78.

Baldus, S., Heeschen, C., Meinertz, T., Zeiher, A. M., Eiserich, J. P., Munzel, T., et al. (2003). Myeloperoxidase serum levels predict risk in patients with acute coronary syndromes. Circulation, 108, $1440-1445$.

Bassand, J. P., Hamm, C. W., Ardissino, D., Boersma, E., Budaj, A., et al. (2007). Guidelines for the diagnosis and treatment of nonST-segment elevation acute coronary syndromes. European Heart Journal, 28, 1598-1660.

Boernsen, K. O., Gatzek, S., \& Imbert, G. (2005). Controlled protein precipitation in combination with chip-based nanospray infusion mass spectrometry. An approach for metabolomics profiling of plasma. Analytical Chemistry, 77(22), 7255-7264.

Brennan, M. L., Penn, M. S., Van Lente, F., Nambi, V., Shishehbor, M. H., Aviles, R. J., et al. (2003). Prognostic value of myeloperoxidase in patients with chest pain. New England Journal of Medicine, 349, 1595-1604.

Chen, J., Zhao, X., Fritsche, J., Yin, P., Schmitt-Kopplin, P., Wang, W., et al. (2008). Practical approach for the identification and isomer elucidation of biomarkers detected in a metabonomic study for the discovery of individuals at risk for diabetes by integrating the chromatographic and mass spectrometric information. Analytical Chemistry, 80(4), 1280-1289.

Chew, D., Allan, R., Aroney, C., \& Sheerin, N. (2005). National data elements for the clinical management of acute coronary syndromes. Medical Journal of Australia, 182(9 Suppl), S1-S16. 
Fiehn, O., Kopka, J., Dörmann, P., Altmann, T., Trethewey, R. N., \& Willmitzer, L. (2000). Metabolite profiling for plant functional genomics. Nature Biotechnology, 18(11), 1157-1161.

García-Pinilla, J. M., Gálvez, J., Cabrera-Bueno, F., Jiménez-Navarro, M., Gómez-Doblas, J. J., Galisteo, M., et al. (2008). Baseline glutathione peroxidase activity affects prognosis after acute coronary síndromes. Texas Heart Institute Journal, 35(3), 262-267.

Hackam, D. G., \& Anand, S. S. (2003). Emerging risk factors for atherosclerotic vascular disease: A critical review of the evidence. Journal of the American Medical Association, 290(7), 932-940.

Han, L. D., Xia, J. F., Liang, Q. L., Wang, Y., Wang, Y. M., Hu, P., et al. (2011). Plasma esterified and non-esterified fatty acids metabolic profiling using gas chromatography-mass spectrometry and its application in the study of diabetic mellitus and diabetic nephropathy. Analytica Chimica Acta, 689(1), 85-91.

Hansson, G. K. (2005). Inflammation, atherosclerosis, and coronary artery disease. New England Journal of Medicine, 352, $1685-1695$.

Heeschen, C., Dimmeler, S., Hamm, C. W., van den Brand, M. J., Boersma, E., Zeiher, A. M., et al. (2003). Soluble CD40 ligand in acute coronary syndromes. New England Journal of Medicine, $348,1104-1111$

Heeschen, C., Hamm, C. W., Bruemmer, J., \& Simoons, M. L. (2000). Predictive value of $\mathrm{C}$-reactive protein and troponin $\mathrm{T}$ in patients with unstable angina: A comparative analysis. CAPTURE Investigators. Chimeric c7E3 AntiPlatelet Therapy in Unstable angina Refractory to standard treatment trial. Journal of the American College of Cardiology, 35, 1535-1542.

James, S. K., Lindahl, B., Siegbahn, A., Stridsberg, M., Venge, P., Armstrong, P., et al. (2003). N-terminal pro-brain natriuretic peptide and other risk markers for the separate prediction of mortality and subsequent myocardial infarction in patients with unstable coronary artery disease: A Global Utilization of Strategies to open occluded arteries (GUSTO)-IV substudy. Circulation, 108, 275-281.

Jiye, A., Gullberg, J., Johansson, A. I., Jonsson, P., Antti, H., Marklund, S. L., et al. (2005). Extraction and GC/MS analysis of the human blood plasma metabolome. Analytical Chemistry, 77, 8086-8094.

Kemsley, E. K., Le Gall, G., Dainty, J. R., Watson, A. D., Harvey, L. J., et al. (2007). Multivariate techniques and their application in nutrition: A metabolomics case study. British Journal of Nutrition, 98(1), 1-14.

Kind, T., Tolstikov, V., Fiehn, O., \& Weiss, R. H. (2007). A comprehensive urinary metabolomic approach for identifying kidney cancer. Analytical Biochemistry, 363(2), 185-195.

Lepage, G., \& Roy, C. C. (1986). Direct transesterification of all classes of lipids in a one-step reaction. Journal of Lipid Research, 27, 114-120.

Lewis, G. D., Wei, R., Liu, E., et al. (2008). Metabolite profiling of blood from individuals undergoing planned myocardial infarction reveals early markers of myocardial injury. Journal of Clinical Investigation, 118(10), 3503-3512.

Libby, P., Ridker, P. M., \& Maseri, A. (2002). Inflammation and atherosclerosis. Circulation, 105(9), 1135-1143.

Mayr, M., Yusuf, S., Weir, G., et al. (2008). Combined metabolomic and proteomic analysis of human atrial fibrillation. Journal of the American College of Cardiology, 51(5), 585-594.
Meyer, M. R., Weber, A. A., \& Maurer, H. H. (2011). A validated GC-MS procedure for fast, simple, and cost-effective quantification of glycols and GHB in human plasma and their identification in urine and plasma developed for emergency toxicology. Analytical and Bioanalytical Chemistry, 400(2), 411-414.

Oliver, S. G., Winson, M. K., Kell, D. B., \& Baganz, F. (1998). Systematic functional analysis of the yeast genome. Trends in Biotechnology, 16(9), 373-378.

Ooi, E. M., Chan, D. T., Watts, G. F., Chan, D. C., Ng, T. W., Dogra, G. K., et al. (2011). Plasma apolipoprotein C-III metabolism in patients with chronic kidney disease. Journal of Lipid Research, 52(4), 794-800.

Paige, L. A., Mitchell, M. W., Krishnan, K. R., Kaddurah-Daouk, R., \& Steffens, D. C. (2007). A preliminary metabolomic analysis of older adults with and without depression. International Journal of Geriatric Psychiatry, 22(5), 418-423.

Pasikanti, K. K., Ho, P. C., \& Chan, E. C. (2008). Gas chromatography/mass spectrometry in metabolic profiling of biological fluids. Journal of Chromatography B, 871(2), 202-211.

Roger, V. L., et al. (2011). Heart disease and stroke statistics-2011 update: A report from the American Heart Association. Circulation, 123(4), e18-e209.

Salek, R. M., Maguire, M. L., Bentley, E., Rubtsov, D. V., Hough, T., Cheeseman, M., et al. (2007). A metabolomic comparison of urinary changes in type 2 diabetes in mouse, rat, and human. Physiological Genomics, 29(2), 99-108.

Shen, Q., Rigor, R. R., Pivetti, C. D., Wu, M. H., \& Yuan, S. Y. (2010). Myosin light chain kinase in microvascular endothelial barrier function. Cardiovascular Research, 87(2), 272-280.

Tweeddale, H., Notley-Mcrobb, L., \& Ferenci, T. (1998). Effect of slow growth on metabolism of Escherichia coli, as revealed by global metabolite pool ('metabolome') analysis. Journal of Bacteriology, 180(19), 5109-5116.

Vallejo, M., García, A., Tuñón, J., García-Martínez, D., Angulo, S., Martin-Ventura, J. L., et al. (2009). Plasma fingerprinting with GC-MS in acute coronary syndrome. Analytical and Bioanalytical Chemistry, 394(6), 1517-1524.

van der Greef, J., Martin, S., Juhasz, P., Adourian, A., Plasterer, T., Verheij, E. R., et al. (2007). The art and practice of systems biology in medicine: Mapping patterns of relationships. Journal of Proteome Research, 6(4), 1540-1559.

van Olffen, R. W., de Bruin, A. M., Vos, M., Staniszewska, A. D., Hamann, J., van Lier, R. A., et al. (2010). CD70-driven chronic immune activation is protective against aterosclerosis. Journal of Innate Immunity, 2(4), 344-352.

Varo, N., de Lemos, J. A., Libby, P., Morrow, D. A., Murphy, S. A., Nuzzo, R., et al. (2003). Soluble CD40L: Risk prediction after acute coronary syndromes. Circulation, 108, 1049-1052.

Waterman, C. L., Kian-Kai, C., \& Griffin, J. L. (2010). Metabolomic strategies to study lipotoxicity in cardiovascular disease. Biochimica et Biophysica Acta, 1801(3), 230-234.

Wilson, P. W. (2008). Progressing from risk factors to omics. Circulation Cardiovascular Genetics, 1(2), 141-146.

Zeng, M., Liang, Y., Li, H., Wang, M., Wang, B., Chen, X., et al. (2010). Plasma metabolic fingerprinting of childhood obesity by GC/MS in conjunction with multivariate statistical analysis. Journal of Pharmaceutical and Biomedical Analysis, 52(2), 265-272. 